Archives de sciences sociales des religions

125 | janvier - mars 2004

Autorités religieuses en islam

\title{
Les Autorités religieuses dans le chiisme duodécimain contemporain
}

Sabrina Mervin

\section{(2) OpenEdition \\ 12 Journals}

Édition électronique

URL : http://journals.openedition.org/assr/1033

DOI : $10.4000 /$ assr. 1033

ISSN : $1777-5825$

Éditeur

Éditions de l'EHESS

Édition imprimée

Date de publication : 1 janvier 2004

Pagination : 63-77

ISBN : 2-222-96741-4

ISSN : 0335-5985

Référence électronique

Sabrina Mervin, "Les Autorités religieuses dans le chiisme duodécimain contemporain », Archives de sciences sociales des religions [En ligne], 125 | janvier - mars 2004, mis en ligne le 22 février 2007,

consulté le 19 avril 2019. URL : http://journals.openedition.org/assr/1033 ; DOI : 10.4000/assr.1033

(c) Archives de sciences sociales des religions 


\section{LES AUTORITÉS RELIGIEUSES DANS LE CHIISME DUODÉCIMAIN CONTEMPORAIN}

En 1890, pour renflouer les caisses de l'État iranien, Nâsir al-Dîn Shah accorda au baron de Reuter, sujet britannique, une concession qui lui garantissait pour cinquante ans le monopole de la culture, de la vente et de l'exportation du tabac. Aux yeux des Iraniens, c'était brader les ressources du pays à des étrangers. La colère gronda, autant dans les cercles cléricaux que dans les milieux bazaris et parmi la population. Le Shah refusa d'entendre les injonctions des oulémas comme de plier devant les manifestations populaires. L'activiste réformiste Jamâl al-Dîn al-Asadâbâdî al-Afghânî(1), qui venait d'être exilé par le souverain, poussa le grand chef religieux chiite de l'époque, un Persan qui résidait à Sâmarrâ', Muhammad Hasan al-Shîrâzî, à réagir. En décembre 1891, celui-ci promulgua une fatwâ déclarant la consommation de tabac illicite. Le boycott fut suivi dans tout le pays, jusqu'au sein même de la cour du Shah, qui dut finalement plier et annuler la fameuse concession (2).

Si le fait constitue un événement historique, traité en tant que tel par les historiens de l'Iran, il est rapporté de façon récurrente par les oulémas chiites, comme un récit exemplaire. Il leur sert à édifier les croyants non seulement sur l'efficacité du boycott, c'est-à-dire de la solidarité nationale, face aux compagnies étrangères, mais aussi sur leur propre rôle dans la société, leur rapport au pouvoir, et l'étendue de leur autorité. Ainsi, par exemple, le clerc libanais Muhsin al-Amîn la raconta à ses amis nationalistes, à Damas, pour les encourager à boycotter la Régie de l'électricité, détenue par des intérêts français, dans la Syrie sous mandat des années 1930. «Le Shah lui-même ne pouvait plus fumer de narguilé, car les femmes du palais les avaient tous fait disparaître, pour obéir à la fatwâ d'al-Shîrâzî... », leur expliqua-t-il (3). Voilà pour démontrer l'efficacité de la solidarité nationale. Quant à l'autorité des clercs, elle est corroborée par l'anecdote suivante qui complète le récit du boycott du tabac et circule encore, de nos jours, dans les milieux cléricaux

(1) Sur ce personnage, cf. KedDIE, $1968 ; 1972$.

(2) Les références à cet événement dans l'histoire contemporaine de l'Iran sont nombreuses. On pourra notamment consulter KeDDIE, 1966 et LAMBTON, 1965.

(3) Voir le témoignage de Adîb al-Safadî, qui était le rédacteur en chef du journal $a l-S h a ' b$ al-dimashqî, dans «al-Adîb al-waflanî », AL-Amîn, 1983, p. 220. 
chiites, par écrit ou oralement. Le directeur de la compagnie britannique voulut s'enquérir sur celui qui entravait son projet, afin de mieux le combattre. Il demanda : «De combien d'hommes dispose-t-il ? ». On lui répondit que le clerc n'avait pas d'armée. « À combien se monte sa fortune? », poursuivit-il. On lui dit qu'il n'avait pas de fortune et vivait très simplement. Le Britannique rétorqua alors que, dans ces conditions, rien ne pouvait être tenté contre cet homme.

C'est non sans fierté que les chiites rapportent cette anecdote. Toute l'autorité de leurs oulémas réside là : d'un mot, ils peuvent braver le pouvoir en place, alors que le pouvoir n'a aucune prise sur eux, puisqu'ils n'ont pas véritablement d'ambition mondaine. On pense à Khomeini défiant le Shah. Avec lui, l'autorité des clercs fut poussée à son paroxysme, avec les conséquences que l'on sait, à savoir l'instauration de la République islamique d'Iran. Certes, la figure du clerc contestataire qui défie le pouvoir n'est pas propre au chiisme, l'histoire de l'islam sunnite en fournit assez d'exemples, d'Ibn Taymiyya (m. 1328) à Abd al-Salâm Yâsîn. Le premier s'en prit aux Mongols, des envahisseurs qui s'étaient emparés du pouvoir et dont il jugeait l'adhésion à l'islam superficielle. En outre, il blâma aussi les soufis qui, au sein de sa propre société, s'adonnaient à des pratiques rituelles non conformes à l'islam rigoriste qu'il prônait (4). Le second est un cheikh marocain contemporain qui osa s'en prendre au roi Hasan II en lui adressant un "conseil » (nasîha). Il s'ancrait ainsi à la fois dans la tradition islamique, qui permet au clerc d'admonester le prince, grâce au principe affirmant la nécessité de commander le bien et d'interdire le mal, et dans une tradition locale de clercs contestataires, connue de son public. Son autorité sur les croyants fut renforcée par le statut d'opposant politique qu'il acquit suite à ce défi, puisqu'il fut astreint à résidence surveillée (5).

Les oulémas chiites n'ont donc pas le monopole de la contestation. Toutefois, on s'accorde à dire que, en général, les oulémas sunnites ont eu tendance, au cours de l'histoire, à légitimer les pouvoirs en place, alors que les chiites ont plutôt adopté une attitude de réserve à leur égard. Au-delà des similitudes évidentes entre les oulémas des deux grandes branches de l'islam, il importe de souligner que les chiites ont des particularismes, autant dans le fondement de leur autorité que dans les modalités de son fonctionnement. Pour résumer et schématiser les processus en œuvre, on peut dire que l'autorité des oulémas sunnites, depuis la centralisation imposée par l'Empire ottoman, l'application des tanzîmât et le contrôle du champ religieux instauré par les États modernes, tend à se réduire. Alors que c'est l'inverse chez les oulémas chiites, qui ont conquis leur pouvoir religieux et assis leur autorité au fil des siècles et, surtout, depuis la seconde moitié du XIX ${ }^{\mathrm{e}}$ siècle. On va voir comment l'épisode de la fatwâ de Shirazi s'insère dans ce mouvement.

Selon la doctrine chiite, les douze imams infaillibles détenaient, de leur vivant, toute forme d'autorité. En effet, les exégètes chiites interprètent différemment des sunnites le verset coranique : «Ô vous qui croyez! Obéissez à Dieu et obéissez au Prophète et à ceux d'entre vous qui détiennent l'autorité » (IV, 59). Pour les sunnites, les détenteurs de l'autorité ( $\hat{u} l \hat{u}$ al-amr) peuvent être les califes et les rois ; pour les chiites, ce sont les imams (6). À l'instar du prophète Muhammad, dont ils transmirent la sunna, les imams étaient, pour leurs fidèles, les guides de la

(4) Sur Ibn Taymiyya, cf. Laoust, 1939.

(5) El Ayadi, 1999, pp. 129-131.

(6) Cité par Momen, 1985, p. 192. 
communauté et les détenteurs des pouvoirs spirituel et temporel (7). C'est pourquoi les califes et les rois qui lui succédèrent furent considérés par les chiites comme des chefs injustes ou des oppresseurs. En outre, dans les doctrines chiites, l'imamat fait partie des fondements de la religion et complète la prophétie. Comme le prophète et sa fille Fâtima, les imams sont tenus pour infaillibles : ils ne commettent pas d'erreur. Par ailleurs, selon les anciens hadith chiites, seuls les imams sont habilités à décliner les normes de la loi sacrée.

Or, selon les doctrines, le douzième imam « disparut », entra en occultation, en 874. Vivant, mais caché, il continua, dans un premier temps, à communiquer ses prescriptions aux fidèles par l'intermédiaire de quatre agents : ce fut la période de l'occultation mineure. Puis, à partir de 941, il cessa d'avoir recours à des agents, et le lien avec ses adeptes fut rompu: on entra dans la période de l'occultation majeure, qui se poursuit actuellement. Les croyances chiites veulent que, au terme de cette période, l'imam attendu, le Mahdî ou Qâ'im, reviendra sur terre pour y restaurer la justice, avant la fin des temps et le jugement dernier.

Ainsi, à partir de 941, la communauté des croyants se retrouva sans guide, aussi bien pour les affaires spirituelles que pour les affaires temporelles. Selon le hadîth chiite, «Toute bannière élevée avant le soulèvement du Qâ'im appartient à un rebelle contre Dieu (tâghût) », tout pouvoir politique était considéré comme inique, illégitime (8). Or, le temps passant, il devenait de plus en plus difficile, pour la communauté, de se passer d'autorité, de référence. Des questions centrales restaient sans réponse : à qui verser les impôts religieux ? Qui peut diriger la prière du vendredi ou lancer le jihâd? Qui détient le pouvoir de statuer, de juger, d'arbitrer les conflits et de faire appliquer les peines, en l'absence de l'imam ? Peu à peu, les oulémas procédèrent à une élaboration doctrinale, afin de s'attribuer les fonctions et les pouvoirs de l'imam, et d'agir en son nom en tant que son délégué (nâ'ib al-imâm) (9). En outre, ils impulsèrent un processus de rationalisation, voire d'idéologisation des doctrines qui s'effectua par étapes successives, au moyen, notamment, de l'introduction de différents concepts clefs. C'est ce long processus qui allait permettre à Khomeini de concevoir sa théorie de wilâyat al-faqîh, "le pouvoir politico-charismatique » ou « guidance » du théologien-juriste, sur laquelle est fondé l'État islamique en Iran (10).

L'ouverture de la «porte de l'ijtihâd», selon l'expression en vigueur, est le volet principal de ce processus. L'exercice de l'ijtihâd consiste à extraire les prescriptions du droit islamique des quatre sources de ce droit, c'est-à-dire, d'une part, des textes sacrés que sont le Coran et la sunna et, d'autre part, d'une série de méthodes et de techniques que l'on regroupe autour des concepts de consensus (ijmâ') et de raisonnement analogique (qiyâs) chez les sunnites, et de raison ('aql) chez les chiites. C'est dire qu'il s'agit d'élaborer les normes de la loi sacrée, la charî'a. Le fait d'exercer l'ijtihâd permet donc de répondre à de nouvelles questions, de réagir à de nouvelles situations et, plus largement, d'introduire le changement dans les normes tout en revenant aux textes. Il s'oppose au taqlîd, le conformisme juridique, consistant à reproduire les normes établies par les anciens.

(7) Cf. Amir-Moezzi, 1992.

(8) Tradition citée par AMIR-MoEZzI, 1997, p. 21, note 65.

(9) Cf. Madelung, 1985, chap. X, «Authority in Twelver Shiism in the absence of the Imam » et SACHEDINA, 1988.

(10) Amir-Moezzi, 1993, p. 79 ; Modarressi, 1984. Voir également Calder, 1982. 
C'est dans ce mouvement d'ouverture que réside une différence essentielle entre l'histoire des doctrines chiites et celle des doctrines sunnites. En effet, même si cette théorie doit être modulée et affinée aujourd'hui, les historiens admettent que le droit islamique sunnite est théoriquement figé, depuis la fixation de ses quatre écoles (malékite, hanéfite, hanbalite et chaféite), au $\mathrm{XI}^{\mathrm{e}}$ siècle. Depuis cette date en effet, les juristes ont eu une large tendance au conformisme, hormis les exceptions notoires de grands savants de l'islam tels Ghazâlî (m. 1111), Ibn Taymiyya (m. 1328) ou Suyûtî (m. 1505). Cette situation a perduré jusqu'au XVIII ${ }^{\mathrm{e}}$ siècle, lorsque quelques oulémas commencèrent à prôner l'exercice de l'ijtihâd. Puis, le mouvement s'est intensifié, à partir de la fin du XIX ${ }^{\mathrm{e}}$ siècle, quand des modernistes revendiquèrent la réouverture de sa porte, afin de mettre l'islam en accord avec l'esprit du siècle. On considère ainsi que c'est le choc avec la culture envahissante de l'Europe qui incita des oulémas réformistes à réagir et à entamer une réflexion sur la question.

Les doctrines chiites connurent le mouvement inverse. Alors que la porte de l'ijtihâd se fermait chez les sunnites, les chiites s'employèrent à l'ouvrir de plus en plus largement et à octroyer des pouvoirs croissants aux oulémas. Al-Tûsî (m. 1067) donna la première impulsion à ce mouvement, que poursuivirent les savants de Hilla Ibn Idrîs (m. 1201), al-Muhaqqiq (m. 1277), al-'Allâma (m. 1325), puis d'autres du Jabal 'Âmil (l'actuel Liban-Sud), Zayn al-Dîn al-'Âmilî dit « le Second Martyr » (m. 1557) et son fils Hasan (m. 1602). Au même moment, en Iran, le souverain safavide Shah Tahsmap nommait le juriste al-Muhaqqiq al-Karakî (m. 1534) représentant de l'imâm (11). Ce courant du chiisme duodécimain, appelé usûlî, se renforça au XIII ${ }^{\mathrm{e}}$ siècle; il devint majoritaire, et les doctrines s'affinèrent (12).

\section{L'institution de la marja'iyya, pilier de l'autorité religieuse}

Un pas décisif fut franchi avec la systématisation de la référence à un savant habilité à exercer l'ijtihâd, en matière de prescriptions religieuses. Elle fut mise en œuvre par Murtadâ al-Ansârî (m. 1864), qui institua la fonction de marja ', « référence à suivre", ou "source d'imitation» pour les croyants (13). Selon cette théorie, les croyants doivent se conformer aux avis émis par le marja', pour tout ce qui concerne les questions afférant au droit islamique : d'où un nouveau sens du terme taqlîd, qui désigne désormais, pour les chiites, le fait de se conformer aux prescriptions d'un marja ' vivant, et non pas, comme c'est le cas chez les sunnites, de se conformer aux écrits des anciens oulémas d'une école juridique donnée. Les prescriptions, parallèlement, s'étaient élargies à tous les domaines de la vie sociale et politique. Ainsi les oulémas pouvaient-ils s'arroger certaines fonctions de l'imam, comme celle de déclarer le jihâd: ce que fit Ja'far Kâshif al-Ghitâ' (m. 1812), lorsqu'il autorisa Fath 'Alî Shah à mener la guerre sainte contre les

(11) Cf. les articles de l'Encyclopédie de l'islam (2e éd.) : "Mardja'i taklîd », «Mudjtahid ».

(12) Cf. Cole, 1985.

(13) Cole, 1983 ; Kazemi Moussavi, 1985. 
Russes (14). Si, par ce geste, le clerc cautionna la politique du prince, d'autres cessèrent ensuite de composer avec le pouvoir, quitte à s'opposer à lui. La fatwâ que promulgua Muhammad Hasan al-Shîrâzî fut une première étape. Après cela, des clercs s'investirent dans les affaires politiques et, notamment, participèrent au mouvement constitutionnaliste (1906-1911) visant à restreindre le pouvoir du Shah.

Après les travaux d'al-Ansârî, d'autres oulémas précisèrent la doctrine, quant aux modalités du taqlîd et aux critères de choix du marja', et mirent en place le fonctionnement de l'institution. Plus tard, des écrits tendirent à montrer qu'elle datait des débuts du chiisme et dressèrent des listes de marja', à partir des plus anciens. Même si l'idée a été reprise par des chercheurs, il s'agit bien là d'une « tradition inventée » (15).

Selon cette doctrine, tout croyant doit suivre les prescriptions d'un marja', énoncées par celui-ci dans un traité pratique de droit islamique, ainsi qu'à ses fatwâ. S'il opte pour un marja' selon des règles établies, elles ne sont pas contraignantes et son choix s'opère donc, au bout du compte, en toute liberté. Les ancrages ethniques, claniques, locaux et familiaux constituent bien évidemment des facteurs influents, mais pas forcément décisifs. En outre, il arrive que des adeptes d'un marja' en suivent un autre, pour certaines questions : ainsi, par exemple, dans les années 1980, bon nombre de chiites suivaient Kho'i (m. 1992) pour les questions religieuses classiques, et Khomeini (m. 1989) pour les affaires politiques. Kho'i étant par ailleurs très rigoriste en matière de voile, puisqu'il prescrivait aux femmes de se cacher le visage, il leur laissait le loisir de suivre un autre marja', sur ce point précis ; certaines se référaient donc à Khomeini en la matière (16). Enfin, le croyant doit régler ses impôts religieux au marja ' qu'il suit. Ainsi, il lui verse la zakât, un impôt commun aux grandes branches de l'islam, mais aussi le khums, spécifique au chiisme, se montant au cinquième de ce qui lui reste lorsqu'il a dépensé ce qu'il lui faut pour vivre.

Avec l'instauration du système de taqlîd, un lien direct est donc établi entre le croyant et un clerc de haut rang auquel il se réfère pour suivre les préceptes de la loi, et ainsi assurer son salut. C'est l'une des raisons pour lesquelles on parle parfois de " clergé » concernant les autorités religieuses chiites qui, en outre, sont organisées en une manière de hiérarchie. Toutefois, celle-ci est très différente de la hiérarchie chrétienne, à laquelle on la compare parfois, et même du système sunnite mis en place par les Ottomans, dont se sont inspirés les États musulmans modernes.

Il n'y a aucune procédure de désignation formelle du marja '; celui-ci n'est ni élu, ni désigné : on dit qu'il « émerge ». En fait, tout se joue à trois niveaux : celui des cercles des clercs de haut rang, celui des milieux commerçants et financiers influents, et, in fine, au niveau des croyants qui entérinent, ou non, les avis des précédents. Ajoutons à cela l'influence éventuelle de l'État, sur laquelle nous reviendrons. Le marja' doit, d'abord, être reconnu comme le plus savant de son temps et jouir d'une grande réputation dans l'enseignement des sciences religieuses ; c'est le premier critère de sélection. Ensuite, il doit faire preuve de piété et de probité morale, et être capable de percevoir les impôts et de les employer à subvenir aux besoins des étudiants en sciences religieuses. Voilà donc les

(14) LAMBTON, 1970.

(15) Abdul-Jabar, 2002, pp. 61-62; Mervin, 2000, p. 124.

(16) WALBridge, 2001 , p. 240 
principales qualités requises pour prétendre à la fonction, qui s'ajoutent aux critères de bases, à savoir : être un homme, de naissance légitime, d’âge mûr, et doué d'intelligence.

La première condition à remplir pour devenir marja', être «le plus savant » en sciences religieuses, est difficile à apprécier. Aussi, des critères plus ou moins objectifs ont été peu à peu mis en œuvre. Avant tout, il faut compter parmi les oulémas de haut niveau, dûment habilités à exercer l'ijtihâd par leurs maîtres, et donc détenteurs de certificats l'attestant. Dans les cercles de ces savants, appelés mujtahid, il faut être reconnu comme l'un des meilleurs. Ce qui ne peut advenir à un jeune clerc, mais requiert âge et expérience. En outre, la réputation du candidat se «mesure" au nombre des étudiants qui suivent son enseignement, et à la manière dont ils le reçoivent : s'ils prennent le soin de noter précisément les cours du maître, et de les publier, cela ne lui donne que plus de poids. C'est donc au sein de l'école en sciences religieuses, la hawza, et dans les cercles de ses pairs que le mujtahid commence à se distinguer. S'il répond aux incitations de son entourage à rédiger un traité pratique de droit islamique, susceptible de devenir un guide pour les croyants, il se pose alors en candidat à la marja 'iyya. Des hommes d'influence (religieuse, sociale, financière) interviennent pour le promouvoir et, au bout de la chaîne, les fidèles vont décider de se référer à lui, ou non : l'allégeance populaire est la dernière étape. C'est ainsi que le marja 'émerge.

Des liens étroits unissent le marja ' aux étudiants en sciences religieuses, donc à la hawza. Ce terme désignait d'ailleurs, à l'origine, le cercle que forment des disciples autour d'un maître, avant que son sens s'étende à l'école religieuse, puis au système d'enseignement qu'elle dispense. La plus ancienne hawza est celle que fonda le cheikh al-Tûsî (m. 1067) à Najaf, l'une des villes saintes du chiisme, aux côtés des autres "seuils sacrés » d'Irak, Karbala, al-Kâzimiyya et Sâmarrâ', qui abritent des mausolées d'imams (17). Centres de pèlerinage, ces villes saintes sont aussi des foyers de savoir, et, particulièrement, Najaf. Celle-ci fut le siège historique de la marja 'iyya. En effet, si d'autres villes saintes ont pu, un temps, voir émerger et abriter un marja', c'est Najaf qui en compta le plus grand nombre (18). Elle est en cela désormais en concurrence avec la ville iranienne de Qom, un ancien foyer de savoir chiite qui fut réactivé, à partir des années 1920. Sa hawza fut ensuite la grande rivale de Najaf, surtout à la faveur de la révolution iranienne qui lui permit de s'étendre et de se moderniser; dans le même temps, la répression qui frappait les chiites, en Irak, provoquait le déclin de celle de Najaf (19). Après la chute du régime de Saddam Hussein, celle-ci devrait renaître, et reprendre sa place. Toutefois, force est de constater que déjà, le marja' le plus suivi aujourd'hui dans le monde chiite est 'Alî Sîstânî qui, s'il est persan d'origine, se réclame néanmoins de la hawza de Najaf, où il réside.

(17) Najaf renferme le mausolée de 'Alî, le premier imam des chiites ; Karbala, celui de Husayn, qui y accomplit son martyre, en 680. D'autres imams ont leurs mausolées à al-Kâzimiyya, à Sâmarrâ', à Médine ou à Machhad, en Iran. Qom a le mausolée de la sœur du huitème imam, Fâtima.

(18) Pour plus de précisions, cf. ABDUL-JABAR, op. cit., p. 81.

(19) Sur la rivalité entre Najaf et Qom, cf. STEWART, 2001. 


\section{Une autorité supra-étatique convoitée}

Au milieu du $\mathrm{XX}^{\mathrm{e}}$ siècle, la centralisation de la marja 'iyya à Najaf entraîna une organisation de l'institution. Le marja' de l'époque, Abû al-Hasan al-Isfahânî, systématisa le recours à des représentants chargés d'assurer la liaison avec ses adeptes résidant dans des zones éloignées. Les marja' ouvrent désormais des bureaux, partout où ils sont représentés auprès des croyants, qui diffusent leurs écrits et leurs fatwâ, et récoltent les impôts religieux. Ce mouvement de centralisation, qui a permis une certaine bureaucratisation de l'institution, a oscillé avec un mouvement de décentralisation, qui a engendré le pluralisme de l'autorité religieuse. En effet si la doctrine présente un marja' a lâ, ou "référence suprême ", dans l'histoire, le consensus autour d'un seul marja 'n'a pas toujours été réalisé, et ils furent parfois plusieurs à assurer la fonction simultanément (20). C'est le cas aujourd'hui, où, après bien des débats et des écrits sur la question, la tendance est non seulement à un pluralisme de fait, mais aussi à un pluralisme souvent affirmé, et revendiqué par les acteurs. Cela, en réaction à la tentative de centralisation iranienne opérée par Ali Khamenei au milieu des années 1990.

Les milieux cléricaux chiites sont très attachés à l'indépendance de la marja 'iyya par rapport à l'État, qui se fonde à la fois sur le caractère transnational du chiisme, et sur l'organisation de l'institution. Son autonomie financière en est un facteur essentiel. Grâce aux impôts religieux qu'il reçoit, le marja' finance les écoles religieuses qui sont sous son autorité, notamment en versant les salaires des professeurs, et en allouant des bourses aux étudiants. Il rémunère par ailleurs ses représentants, et peut rétribuer des clercs. En outre, il fait construire des mosquées, des husayniyya (21), des hôpitaux, des dispensaires, des orphelinats et autres sociétés de bienfaisance. Les fonds investis renforcent ainsi son capital symbolique, à savoir son prestige et son autorité religieuse. En outre, le système permet à la marja 'iyya d'assurer la formation des clercs et d'entretenir une hiérarchie religieuse indépendante - et, ce, même en Iran, malgré les pressions du gouvernement islamique. C'est une grande différence avec le monde sunnite, où les États modernes se sont employés, en ayant recours à différentes stratégies, à contrôler la formation des clercs et leur nomination à des fonctions religieuses officielles (22). Certes, cela n'empêche pas la présence, en parallèle, d'autorités autoproclamées, qui occupent une partie du champ religieux, mais celles-ci ne relèvent alors d'aucune hiérarchie, et ont parfois des statuts précaires. Les milieux cléricaux chiites, à l'inverse, contrôlent et régulent le champ religieux, par le biais de la marja 'iyya. Ce qui n'a pas manqué de susciter des tentatives de mainmise de la part de certains États.

Ainsi, avant la révolution islamique, l'Iran essayait déjà d'influer, d'une certaine manière, sur le choix d'un nouveau marja'. En effet, depuis l'accession à la marja 'iyya de Borûjerdî en 1945, lorsqu'un marja' venait à mourir, le Shah adressait un télégramme de condoléances au grand clerc qu'il voulait voir lui succéder. Aussi les chiites attendaient-ils de voir à qui serait destiné le télégramme,

(20) ABDUl-JABAR, op. cit., p. 80.

(21) Lieux de culte spécifiques au chiisme où se tiennent les séances de déploration à la mémoire de l'imam Husayn, ainsi que des conférences destinées à édifier les fidèles.

(22) Cf. ZEgHAL, 1999. 
pour connaître la position du souverain (23). L'avènement de la république islamique changea radicalement la situation, au moins à l'intérieur de l'Iran, puisque Khomeini assura à la fois la wilâyat al-faqîh, la guidance du théologien-juriste, c'est-à-dire l'exercice du pouvoir politique, et la marja 'iyya, le pouvoir spirituel. Toutefois, il ne l'exerça pas de manière absolue, puisque d'autres marja 'étaient suivis, même en Iran, notamment Kho'i et Montazeri. Pressentant, avant de mourir, que sa succession poserait un sérieux problème, Khomeini révisa sa théorie de wilâyat al-faqîh, de sorte qu'il opéra, de facto, une séparation entre pouvoir religieux et pouvoir politique, même si celui-ci continuait à être tenu par des clercs. C'est d'ailleurs la situation qui prévaut, de facto, dans l'Iran actuel. Ensuite, il nomma à la tête de l'État Ali Khamenei, un clerc qui ne pouvait prétendre à la marja 'iyya car il n'avait pas les qualifications requises en matière de savoir religieux, puisqu'il n'était même pas mujtahid. D'autres que lui furent donc mis en avant par la république islamique (24). Toutefois, en 1995, Khamenei fut déclaré marja'. Face à l'opposition que suscita cette décision, Khamenei déclara qu'il n'était pas candidat à la marja 'iyya en Iran, mais pour le reste du monde chiite. Le paradoxe de la situation ne manqua pas de frapper les chiites de l'extérieur, qui se retrouvaient face à une marja 'iyya imposée par l'Iran, alors que les sujets iraniens, eux, gardaient la possibilité de choisir. Ils refusèrent, pour la grande majorité d'entre eux, de se plier à cette politique d'unification et de centralisation. L'Iran dut se rétracter et accepter de voir se développer une marja 'iyya plurielle, qu'il pouvait tenter de circonscrire à l'intérieur, mais incontrôlable, hors de ses frontières. Le cas de Muhammad Husayn Fadl Allâh, au Liban, est très significatif : il ne s'aligna pas sur la direction iranienne, mais s'imposa lui-même comme un marja' indépendant, malgré les pressions qu'il subit (25).

Le régime irakien tenta, lui aussi, de contrôler la marja ‘iyya. Comme Kho'i, puis ses successeurs, lui échappaient totalement, il promut un marja', Muhammad al-Sadr, un clerc de Najaf issu d'une prestigieuse famille d'oulémas. Ce geste fut pris par les chiites, notamment à l'extérieur, pour ce qu'il était : une ingérence dans les affaires de la marja iyya. Toutefois, peu à peu, Muhammad al-Sadr gagna une certaine popularité, à Najaf, et prit ses distances par rapport au régime qui l'avait mis en place. Tant et si bien qu'il en vint à le critiquer publiquement, et le paya du prix de sa vie : après l'avoir assigné à résidence, Saddam Hussein le fit assassiner en février 1999 (26). Depuis, ses partisans sont restés actifs et ont une assise populaire, à Najaf, ainsi qu'une représentation à l'extérieur, notamment à Sayyida Zaynab, en Syrie, où résident de nombreux réfugiés irakiens.

Ainsi, les deux États les plus à même d'avoir mainmise sur la marja 'iyya, et donc sur la hiérarchie religieuse chiite, ne sont pas parvenus à l'accaparer ou à l'instrumentaliser. Reste que le fait de l'abriter confère au moins un certain prestige vis-à-vis des communautés chiites, et apporte une activité économique non négligeable au pays.

L'indépendance de la marja 'iyya par rapport à l'État favorise le caractère transnational du chiisme, puisqu'elle réunit sous une même autorité des réseaux d'adeptes implantés dans différents pays. Ce qui n'empêche ni l'ancrage des chiites

(23) MARTIN, 1993, p. 243.

(24) WALBRIDGe, 2001, pp. 234-237.

(25) Cf. AL-SHÂKHÛRÎ, 1998.

(26) Walbridge, op. cit., p. 238 ; Martin, op. cit., p. 252. Voir aussi RA'Ûf, 1999. 
dans leur région d'origine, ni leur attachement à une identité nationale. Bien plus, le mouvement d'intégration des communautés chiites dans les États dont ils sont ressortissants a tendance à s'intensifier. Au plan de l'organisation des affaires religieuses, le Liban a été le premier pays (l'Iran n'étant pas à prendre en compte) à permettre à la communauté chiite d'être représentée par une institution propre, le Conseil supérieur islamique chiite. Le clerc qui parvint à obtenir sa fondation en 1967, Mûsâ al-Sadr, était d'ailleurs un iranien récemment installé au Liban. La création de ce Conseil fut un pas de plus vers la reconnaissance d'une hiérarchie religieuse chiite interne, qui se charge de régler les affaires de la cléricature libanaise, tout en entretenant des liens, en parallèle, avec la hiérarchie supra-étatique issue du système de la marja iyya (27).

Enfin, l'indépendance de la marja 'iyya par rapport à l'État implique celle de la hawza et de la formation des clercs. La réforme du système de l'enseignement religieux supérieur chiite n'est donc pas comparable à celle que connurent les grands établissements sunnites comme al-Azhar, qui se modernisèrent sous la pression de l'État. Elle se fit plus lentement, suite aux initiatives individuelles et dispersées de grands mujtahid ou de marja: Les nouvelles générations de clercs chiites ainsi formés ne furent donc pas promues par l'État, mais par la hiérarchie religieuse. Ce qui explique, en partie, que les mouvements islamiques chiites sont issus de cette hiérarchie, contrairement à la tendance prévalant dans les mouvements sunnites.

\section{Les oulémas chiites : modèles classiques et nouveaux acteurs}

Rappelons que les fondateurs des mouvements islamistes sunnites, Hasan al-Bannâ, Sayyid Qutb, Mawdûdî, étaient de petits intellectuels, autodidactes en matière de sciences religieuses. Bon nombre de leurs successeurs ont ce type de profil, même s'ils furent rejoints, ensuite, par des clercs. Cela ne fut pas le cas des dirigeants des mouvements chiites : une autorité religieuse autoproclamée avait peu de chance de se faire entendre sans la reconnaissance des milieux cléricaux. Or, s'ils sont théoriquement ouverts à tout nouveau venu, à condition qu'il fasse ses preuves en matière de sciences religieuses et qu'il se distingue par l'exercice de l'ijtihâd, on constate que ceux-ci sont peu nombreux à sortir du rang. Les grands clercs chiites forment une aristocratie religieuse qui se reproduit à travers la hawza. La plupart d'entre eux appartiennent à des «familles de sciences » dont sont issus les oulémas, depuis plusieurs générations. Ces familles tirent leur légitimité d'une ascendance prestigieuse, un savant qui a marqué l'histoire du chiisme, par exemple. Bien plus, certaines proclament être des lignages descendant du prophète Muhammad, généalogie dûment certifiée par des autorités religieuses à l'appui. Ce sont les familles de sayyid, dont les membres portent ce titre honorifique et se coiffent d'un turban noir marquant leur ascendance (28). Si, dans les milieux sunnites, on a pu observer que suivre un enseignement en sciences religieuses pour se former à la cléricature était une stratégie d'ascension sociale, chez les chiites, c'est un moyen de reproduction de l'élite religieuse (29).

(27) Sur le processus d'intégration des chiites libanais, cf. Mervin, 2000, chap. IV et sur Mûsâ al-Sadr, 2002.

(28) Pour plus de détails, Mervin, 2000, chap. III, «Qu'est ce qu'un 'âlim ? ».

(29) Voir à ce sujet les résultats de l'enquête menée par KHURI, 1990, pp. $170 ; 182$. 
Cette élite religieuse assure par ailleurs sa cohésion par une relative endogamie, en prenant des femmes soit dans les familles de notables, soit dans les familles d'oulémas. Ainsi, certaines peuvent rester alliées par des séries d'intermariage, sur plusieurs générations. En outre, les affinités se renforcent par le biais des liens qui se tissent entre le maître et son disciple, et entre des étudiants qui poursuivent ensemble, pendant de longues années, un cursus ardu. Fait notable, ces familles religieuses sont souvent transnationales. La famille al-Sadr, par exemple, qui est originaire du Liban-Sud (le lignage apparenté y porte aujourd'hui le nom de Sharaf al-Dîn), a une branche en Irak, et une autre en Iran.

Les dirigeants des mouvements islamiques « révolutionnaires » étaient issus de ces grandes familles. Ainsi de Muhammad Bâqir al-Sadr, leader du mouvement islamique irakien, exécuté en 1980 par le régime ; de son cousin Mûsâ al-Sadr qui, venu d'Iran, s'installa au Liban où il fut l'artisan du " réveil » de la communauté chiite avant de disparaître mystérieusement lors d'un voyage en Libye, en 1978 ; ou bien de Khomeini, le leader de la révolution iranienne (m. 1989). En outre, c'étaient de grands clercs, des mujtahid; Muhammad Bâqir al-Sadr et Khomeini furent même des marja'. Ils avaient le profil attendu pour assurer cette fonction, mais tinrent un discours novateur en la matière. Le premier appela à une rénovation de la fonction de marja', le second y apporta une redéfinition. Quant à Mûsâ al-Sadr, il fut parmi les premiers à avoir reçu une double formation, universitaire, à la faculté de droit de l'université de Téhéran, et religieuse, dans les écoles de Qom et de Najaf. D'autres oulémas iraniens, qui participèrent activement à la révolution, tel Muhammad Beheshti, assassiné à ses débuts, en 1981, avaient ce nouveau profil.

Le modèle classique a cependant perduré. Le grand marja' Kho'i, qui fut le rival de Khomeini, était non seulement, comme lui, issu d'une famille religieuse, mais il exerça ses fonctions sans vraiment s'intéresser aux questions qui constituaient le centre des débats dans bien des cercles cléricaux, comme le rapport du religieux et du politique, la nécessité de modernisation du discours religieux et des institutions, etc. Alî Sîstânî, l'actuel grand marja' qui se pose comme son successeur, a la même vision, classique, de sa fonction. On dit même qu'il ne lit pas la presse, ni ne se penche sur les affaires du monde (30). Le fait est d'autant plus remarquable que Sîstânî est le marja' le plus suivi dans le monde chiite.

Pour autant, d'autres se positionnent différemment, et le revendiquent. C'est le cas de Muhammad Husayn Fadl Allâh qui, s'il relève du modèle classique du clerc chiite pour ce qui est de son origine et de sa formation, a participé au mouvement islamique à partir des années 1960, et tient aujourd'hui un discours résolument réformiste et moderniste. Il clame la nécessité de revoir les qualifications nécessaires au marja': pour lui, celui-ci doit impérativement, aujourd'hui, avoir une bonne connaissance des affaires mondaines. On voit donc que deux conceptions du rôle et de la fonction de marja' se dégagent : le modèle classique du marja ' attentiste, apolitique et traditionnel ; le paradigme du nouveau marja', qui s'implique en politique, se veut à l'écoute des changements sociaux, et s'engage dans la réforme des idées et des institutions religieuses.

(30) WALBRIDGE, op. cit., p. 237. Des proches de Sistani démentent cependant cette affirmation, alors même que Ali Sîstânî s'engage dans la défense des règles de la démocratie pour la reconstruction de l'Irak - Entretien avec Ghanem Jawad, fondation Khoei, Londres, 6.12.03. 
Le profil des générations montantes de oulémas est par ailleurs en train de changer. Si les grands clercs en place aujourd'hui, dans le monde arabe notamment, appartiennent encore aux anciennes familles de science, et ont été formés à la hawza, de nouveaux acteurs religieux entrent en scène, suite à l'influence de l'Iran post-révolutionnaire. D'abord, l'Iran a favorisé la vulgarisation du savoir religieux, en ouvrant largement ses écoles, de Qom et de Machhad, notamment, aux Iraniens et aux étrangers. Cela provoqua un décloisonnement des milieux cléricaux, qui ont été investis par des étudiants en sciences religieuses issus de familles non spécialisées dans la cléricature. En outre, le suivi d'un double cursus, universitaire et religieux, s'est développé ; 1'Iran a favorisé le phénomène en instaurant un système d'équivalence entre les deux. En Iran, aujourd'hui déjà, bon nombre de clercs sont aussi des universitaires, parfois titulaires d'un doctorat; à l'inverse, on trouve des intellectuels issus du système universitaire qui, par ailleurs, ont reçu un enseignement religieux. On a donc des clercs intellectuels et des intellectuels religieux. Ce phénomène s'étend aux milieux chiites libanais et irakiens, par exemple. Dans la hawza placée sous les auspices de Muhammad Husayn Fadl Allâh, à Beyrouth, la moitié des étudiants suit en parallèle un cursus universitaire. Le directeur de l'école lui-même est un clerc, et il est docteur en philosophie islamique.

\section{Un « désordre organisé »}

L'enseignement dispensé dans les écoles religieuses chiites a été réformé, depuis les premières critiques formulées par des clercs contre leur manque d'organisation et de pédagogie, à la fin des années 1920. Des oulémas parvinrent progressivement à introduire des sciences profanes au cursus religieux, et à rationaliser le fonctionnement des cours, par la mise en place de programmes, de classes, d'horaires fixes. Ce ne fut pas sans difficulté. En effet, le système de la hawza reposait entièrement sur la totale liberté de l'étudiant de choisir un maître, et sur le lien qui s'établissait entre maître et disciple. Une organisation sur le modèle des écoles profanes, modernes, allait contre ces principes. C'est pourquoi la réforme de la hawza rencontra beaucoup de résistance dans les milieux cléricaux. Aujourd'hui encore, alors que la plupart des écoles fonctionnent sur un mode réformé (plus ou moins), des oulémas évoquent ces principes comme les garants de la formation d'une véritable élite religieuse, non sans nostalgie (31).

Un autre principe cardinal, l'indépendance de la hawza, est menacé par tout processus de réforme allant dans le sens de l'organisation des écoles et de leur bureaucratisation. L'histoire récente en fournit un exemple frappant, celui de Kulliyyat al-fiqh, un collège de droit islamique fondé à Najaf, en 1958. Après des années d'hésitation due à l'opposition de leurs pairs, un groupe de clercs réformistes, Muhammad Ridâ al-Muzaffar en tête, ouvrit ce collège dont l'organisation était calquée sur celle des établissements modernes. Bien plus, il fut reconnu par l'État, puis rattaché à l'université irakienne. Tant et si bien que, lors de la répression contre les chiites de 1991, le gouvernement le ferma.

C'est pourquoi, entre la nécessité de moderniser et l'attachement à un système souple, ne reposant que sur l'autorité d'un marja', les chiites oscillent sans cesse. Il

(31) Sur le système traditionnel, cf. Mervin, 1995 ; sur la réforme des écoles, Mervin, 2000, pp. 212-225; 2003 ; LiTVAK, 1998. 
en est de même pour la réforme de la marja 'iyya. En fait, l'institution, relativement jeune, est en perpétuel développement et fait l'objet de nombreuses discussions, entre grands clercs. Néanmoins, elle reste entièrement centrée sur la personne du marja' et sur son charisme ; celui-ci ne pouvant se charger des fonctions administratives, il délègue ses proches, surtout ses fils et ses gendres, qui s'occupent par exemple des investissements financiers ou de la gestion des fondations. Tout cela fonctionne donc sur un mode patriarcal et informel. À plusieurs reprises, il a été question de réformer la marja 'iyya pour mieux l'organiser et la rationaliser. D'une part, il s'agissait de revoir les modalités de désignation du marja 'afin de les formaliser, voire d'instaurer une direction collégiale ; d'autre part, de mettre en place une structure susceptible d'administrer l'institution et de la pérenniser après la mort du marja' (32). Autrement dit, c'était permettre un processus de routinisation. Or, ces différentes tentatives restèrent lettres mortes, les chiites n'étant pas prêts à se doter d'une institution qui serait comparable au Vatican, avec un chef élu et une hiérarchie pyramidale, même si le modèle est tentant, pour certains. Un embryon de hiérarchie a été mis en place en Iran, notamment par la transformation de titres honorifiques, hujjat al-islâm, ayatollâh, en grades fondés sur le degré de savoir, mais elle est assez floue.

L'autorité religieuse chiite demeure donc dans le "désordre organisé », pour reprendre une expression utilisée par les clercs et les étudiants de la hawza (33). Cela ne signifie pas qu'elle est incontrôlée. Si la marja 'iyya obéit toujours à des règles écrites de base, publiées dans des ouvrages, elle est aussi soumise à des usages et à des règles morales, plus ou moins implicites, discutées dans les cercles cléricaux. Ainsi, par exemple, lorsque les fils d'un marja ' prennent trop de libertés ou veulent s'arroger des fonctions revenant à leur père, on rappelle que la marja 'iyya ne s'hérite pas, et que les institutions fondées par le marja' ne reviennent pas à ses proches, mais au marja' suivant. Le débat doctrinal ainsi que l'autocritique du corps des oulémas permettent de réguler l'institution de la marja'iyya, et laissent la porte ouverte à tout changement d'un système voulu souple, labile, évolutif.

Sabrina MERVIN CNRS

(32) LAMBton, 1964 ; Martin, op. cit., pp. 263-264 ; ABDUL-JABAR, op. cit., pp. 82-84; Al-Hasanî, 1993.

(33) Hânî Fahs, réponses écrites à un questionnaire, Beyrouth, 1999. 


\section{BIBLIOGRAPHIE}

ABDUL-JABAR Faleh, Ayatollahs, Sufis and Ideologues. State, Religion and Social Movements in Iraq, Londres, Saqi Books, 2002.

AL-AMÎN Muhsin, Sîratuhu bi-qalamihi wa aqlâm âkharîn, (textes réunis par Hasan al-Amîn), s. 1., 1983.

AMIR-MOEZZI Mohammad Ali, Le Guide divin dans le Shi'isme originel. Aux sources de l'ésotérisme en islam, Paris, Verdier, 1992.

AMIR-MOEZZI Mohammad Ali, «Réflexion sur une évolution du shi'isme duodécimain : tradition et idéologisation », in Évelyne PATLAGEAN, Alain LE BOULLUEC, éds., Les retours aux écritures, Louvain-Paris, Peeters, 1993, pp. 63-82.

AMIR-MOEZZI Mohammad Ali, «Remarques sur les critères d'authenticité du hadîth et l'autorité du juriste dans le shi'isme imâmite », Studia Islamica, 85/1, février 1997, pp. 5-39.

CALDER Norman, «Accommodation and Revolution in Imami Shi'i Jurisprudence: Khumayni and the Classical Tradition », Middle Eastern Studies, 18/1, janvier 1982, pp. 3-20.

COLE Juan R., «Imami Jurisprudence and the Role of the Ulama: Mortaza Ansari on Emulating the Supreme Exemplar ", in Nikki KEDDIE, ed., Religion and Politics in Iran. Shi'ism from Quietism to Revolution, New Haven-Londres, Yale University Press, 1983, pp. 33-46.

COLE Juan R., «Shi'i Clerics in Iraq and Iran, 1722-1780: The Akhbari-Usuli Conflict Reconsidered », Iranian Studies, 18/1, hiver 1985, pp. 18-23.

El AYADI Mohammed, «Abdessalam Yassine ou le poids des paradigmes dans le parcours d'un nouveau clerc », Parcours d'intellectuels maghrébins, Paris, Karthala, 1999, pp. 129-163.

AL-HASANÎ Salîm, al-Ma'âlim al-jadîda li-l-marja 'iyya al-shî’iyya. Dirâsa wa hiwâr ma'a âyat Allâh al-sayyid Muhammad Husayn Fadl Allâh, Beyrouth, Dâr al-Malâk, 1993.

KAZEMI MOUSSAVI Ahmad, "The Establishment of the Position of marja'iyyat-i Taqlid in the Twelver-Shi'i Community », Iranian Studies, 28/1, 1985, pp. 35-51.

KEDDIE Nikki R., Religion and Rebellion in Iran: The Tobacco Protest of 1891-1892, Londres, Frank Cass, 1966.

KEDDIE Nikki R., An Islamic Response to Imperialism: Political and Religious Writings of Sayyid Jamâl Ad-Dîn "Al-Afghân̂̂", Los Angeles, University of California Press, 1968.

KEDDIE Nikki R., Sayyid Jamâl Ad-Dîn "Al-Afghan̂̀”: A Political Biography, Berkeley-Los Angeles-Londres, University of California Press, 1972.

KHURI Fuad, "The Ulama: A Comparative Study of Sunni and Shi'a Religious Officials », Middle Easten Studies, vol. 23, no 3, juillet 1987, pp. 291-312.

KHURI Fuad, Imams and Emirs, State, Religion and Sects in Islam, Londres, Saqi Books, 1990.

LAOUST Henri, Essai sur les doctrines sociales et politiques de Takî-d-Dîn Ahmad b. Taimîya, Le Caire, IFAO, 1939.

LAMBTON Ann K. S., «A Reconsideration of the Position of the marja' al-taqlid and the Religious Institution », Studia Islamica, 20, 1964, pp. 115-135.

LAMBTON Ann K. S., «The Tobacco Regie: Prelude to Revolution », Studia Islamica, XXII, 1965, pp. 119-157 et XXIII, pp. 71-90.

LAMBTON Ann K. S., " A Nineteenth Century View of Jihâd », Studia Islamica, 32, 1970, pp. 181-192.

LITVAK Meir, Shi'i Scholars of Nineteenth-Century Iraq: The 'Ulama' of Najaf and Karbala', Cambridge, Cambridge University Press, 1998.

MADELUNG Wilferd, Religious Schools and Sects in Medieval Islam, Londres, Variorum Reprints, 1985.

MARTIN Pierre, «La direction religieuse chiite dans une zone de turbulences», Peuples Méditerranéens, Statégies II, no 64-65, juillet-décembre 1993, pp. 241-264.

MERVIN Sabrina, « La quête du savoir à Najaf. Les études religieuses chez les chi'ites imâmites de la fin du XIX ${ }^{\mathrm{e}}$ siècle à $1960 »$, Studia Islamica, 81, 1995, pp. 165-185.

MERVIN Sabrina, Un réformisme chiite. Ulémas et lettrés du Gabal Amil (actuel Liban-Sud) de la fin de l'Empire ottoman à l'indépendance du Liban, Karthala-CERMOC-IFEAD, Paris 2000.

MERVIN Sabrina, "Les yeux de Mûsâ al-Sadr », in Catherine MAYEUR, dir., Saints et héros du Moyen-Orient contemporain, Paris, Maisonneuve et Larose, 2002, pp. 285-300. 
MERVIN Sabrina, «La hawza à l'épreuve du siècle : la réforme de l'enseignement religieux supérieur chiite de 1909 à nos jours ", in Maher AL-CHARIF, Salam KAWAKIBI, Le courant réformiste musulman et sa réception dans les sociétés arabes, PIFD 208, Damas, IFPO, 2004, pp. 69-84.

MODARRESSI Hossein, "Rationalism and Traditionalism in Shî’̂̂ Jurisprudence : A Preliminary Survey », Studia Islamica, LIX, 1984, pp. 141-158.

MOMEN Moojan, An Introduction to Shi'i Islam, New Haven-Londres, Yale University Press, 1985.

RA'ÛF 'Âdil, Muhammad Muhammad Sâdiq al-Sadr, marja 'iyyat al-maydân, mashrû'uhu al-taghyyurî wa waqâ'i' al-ightiyâl, Damas, al-markaz al-'iraqî li-1-i'lâm wa al-dirâsât, 1999.

RICHARD Yann, "Le rôle du clergé : tendances contradictoires du chi'isme iranien contemporain », Archives des sciences sociales des religions, 55/1, 1983, pp. 5-27.

SACHEDINA Abdulaziz, The Just Ruler in Shi'ite Islam. The Comprehensive Authority of the Jurist in Imamite Jurisprudence, New York, Oxford University Press, 1988.

AL-SHÂKHÛRÎ al-Bahrânî, Ja'far, marja 'iyyat al-marhala wa ghubâr al-taghyîr, Beyrouth, Dâr al-amîr/Dâr al-rasûl al-akram, 1998.

STEWART Devin J., "The portrayal of en Academic Rivalry. Najaf and Qom in the Writings and Speeches of Khomeini, 1964-1978 », in Linda WALBRIDGE, ed., The Most Learned of the Shi 'a. The Institution of the marja' Taqlid, New York, Oxford University Press, 2001, pp. 216-229.

WALBRIDGE, Linda, "The Counterreformation. Becoming a marja“ in the Modern world », in Linda WALBRIDGE, ed., The Most Learned of the Shi'a. The Institution of the marja' Taqlid, Oxford, Oxford University Press, 2001, pp. 230-246.

ZAMAN, Muhammad Qasim, The Ulama in Contemporary Islam. Custodians of change, Princeton, Princeton University Press, 2002.

ZEGHAL Malika, «État et marché des biens religieux en Islam », Critique internationale, n 5 , automne 1999, pp. 75-95.

ZEGHAL Malika, « Religion and Politics in Egypt: the ulema of al-Azhar, radical islam and the State (1952-1994)», IJMES, 31, 1999, pp. 371-399. 
Résumé

Face aux pouvoirs politiques en place, les oulémas chiites ont adopté une attitude de réserve, voire de contestation, car ils considèrent que seul l'imam est à même d'exercer l'autorité spirituelle et politique sur la communauté des croyants. À partir du XI siècle, ils élaborèrent des doctrines leur permettant d'élargir leur autorité religieuse, jusqu'à l'institution de la marja 'iyya. Depuis la fin du XIX $X^{e}$ siècle, celle-ci s'est développée et organisée en une autorité religieuse supra-étatique et transnationale, plus ou moins centralisée, dont l'indépendance par rapport à l'Etat repose sur l'autonomie financière des clercs. Ceux-ci constituent une élite religieuse soucieuse d'assurer sa cohésion et sa reproduction. Cependant, la vulgarisation du savoir, promue notamment par la République islamique d'Iran, a amené de nouveaux acteurs. Quant à la marja 'iyya, elle fait l'objet de débats qui tendent à l'organiser.

\section{Abstract}

In their relationship with the political powers in place, Shiite ulemas have adopted an attitude of reserve, or even of protest, because they consider that the Iman is the only one who can exercise spiritual and political authority over the community of believers. In the $11^{\text {th }}$ century, they started to develop doctrines that allowed them to widen their religious authority. This culminated in the creation of the marja iyya, which, since the end of the $19^{\text {th }}$ century, has developed into a more or less centralized organization that is above the State and transnational, and whose independence vis à vis the State rests in the financial autonomy of the clerics. The latter constitute a religious elite preoccupied with remaining cohesive and reproducing. However, the popularization of knowledge promoted, in part, by the Islamic Republic of Iran, has introduced new players. As for the marja'iyya, it is the subject to debates trying to organize it.

\section{Resumen}

Frente a los poderes políticos constituidos, los ulemas chiítas han adoptado una actitud de reserva, cuando no de contestación, dado que ellos consideran que sólo el Imam debe ejercer la autoridad espiritual y politica sobre la comunidad de los creyentes. A partir del siglo XI, ellos elaboran doctrinas que les permiten ampliar su autoridad religiosa, hasta la institución de la marja 'iyya. Desde fin del siglo XIX, ésta se desarrolla y organiza en una autoridad religiosa supraestatal y transnacional, más o menos centralizada, cuya independencia respecto del Estado reposa en la autonomía financiera de los clérigos. Éstos constituyen una élite religiosa atenta a asegurar su cohesión y su reproducción. Sin embargo, la vulgarización del saber, promovida especialmente por la República Islámica de Irán, ha presentado actores nuevos. Cuanto a la marja iyya, ésta es objeto de debates que tienden a organizarla. 
